INPLASY

PROTOCOL

To cite: Wang et al. Efficacy and safety of daratumumab in the treatment of multiple myeloma: a systematic review and meta analysis. Inplasy protocol 202150092. doi: 10.37766/inplasy2021.5.0092

Received: 25 May 2021

Published: 25 May 2021

Corresponding author: Yin Wang

wangyin19@|zu.edu.cn

Author Affiliation:

Department of Haematology, Lanzhou University Second Hospital, Lanzhou, Gansu

Province, China.

Support: There is no fund support.

Review Stage at time of this submission: The review has not yet started.

Conflicts of interest:

None declared.

\section{Efficacy and safety of daratumumab in the treatment of multiple myeloma: a systematic review and meta analysis}

Wang, Y1; Li, YQ2; Chai, Y³.

Review question / Objective: The purpose of this Meta analysis is to systematically evaluate the efficacy and safety of Dara in patients with $\mathrm{MM}$, in order to provide more reference basis for clinical drug selection.

Condition being studied: Multiple myeloma(MM) is a malignant disease with abnormal proliferation of clonal plasma cells, which is still incurable. With the clinical application of various new drugs, the survival and prognosis of MM patients has been significantly improved, but most MM patients still face the risk of recurrence. Therefore, it is very important to introduce more effective drugs into the treatment strategy of MM patients.

Information sources: We will search articles in four electronic database including PubMed, Web of Science, ScienceDirect and The Cochrane Library. All the English publications until 1 June 2021 will be searched without any restriction of countries or article type. Reference list of all selected articles will independently screened to identify additional studies left out in the initial search.

INPLASY registration number: This protocol was registered with the International Platform of Registered Systematic Review and Meta-Analysis Protocols (INPLASY) on 25 May 2021 and was last updated on 25 May 2021 (registration number INPLASY202150092).

\section{INTRODUCTION}

Review question / Objective: The purpose of this Meta analysis is to systematically evaluate the efficacy and safety of Dara in patients with $\mathrm{MM}$, in order to provide more reference basis for clinical drug selection.
Condition being studied: Multiple myeloma(MM) is a malignant disease with abnormal proliferation of clonal plasma cells, which is still incurable. With the clinical application of various new drugs, the survival and prognosis of MM patients 
has been significantly improved, but most MM patients still face the risk of recurrence. Therefore, it is very important to introduce more effective drugs into the treatment strategy of MM patients.

\section{METHODS}

Participant or population: Patients with multiple myeloma.

Intervention: The intervention group was given a treatment plan including Daratumumab, and the specific treatment plan was not limited.

Comparator: The control group was a treatment scheme without Daratumumab, and the specific scheme was not limited.

Study designs to be included: Randomized controlled trials (RCTs).

Eligibility criteria: Inclusion criteria: patients with newly diagnosed multiple myeloma (NDMM) or relapsed refractory Multiple myeloma (RRMM) treated with Daratumumab.

Information sources: We will search articles in four electronic database including PubMed, Web of Science, ScienceDirect and The Cochrane Library. All the English publications until 1 June 2021 will be searched without any restriction of countries or article type. Reference list of all selected articles will independently screened to identify additional studies left out in the initial search.

Main outcome(s): Overall response rate (ORR), complete response or better ( $\geq \mathrm{CR}$ ), incidences of neutropenia ( $\geq$ grade 3 ), thrombocytopenia ( $\geq$ grade 3 ) and pneumonia.

Additional outcome(s): The incidence of secondary tumor.

Quality assessment / Risk of bias analysis: Using GRADE to evaluate the quality of evidence.
Strategy of data synthesis: RevMan 5.4 software was used for Meta analysis. The odds ratio (OR) was used as the effect analysis statistic of the two classified variables, and its $95 \% \mathrm{Cl}$ was provided. The heterogeneity among the results was analyzed by $x 2$ test, and the heterogeneity was quantitatively judged by $\mathbf{I}^{2}$. If there is no statistical heterogeneity among the results of each study, the fixed effect model is used for Meta analysis. If there is statistical heterogeneity among the results, the source of heterogeneity is further analyzed. After excluding the obvious clinical heterogeneity, the random effect model is used for Meta analysis. The obvious clinical heterogeneity was treated by subgroup analysis or sensitivity analysis, or only descriptive analysis. The funnel chart was drawn by RevMan5.4 software to test the publication bias. Based on the Bayesian hierarchical model, the networkMeta analysis of the outcome index was carried out by using ADDIS software. The first iteration is set to 50000 . The convergence of the network Meta analysis is tested by the potential scale reduction parameter (PSRF). If the PSRF is closer to 1 , the better the convergence efficiency is, the higher the credibility of the model analysis conclusion is. The concordance model was used in the analysis of networkMeta. The rank probability ranking chart was used to show the ranking of the outcome indicators of each intervention.

Subgroup analysis: Subgroup analysis will be performed in group of newly diagnosed multiple myeloma (NDMM) and recurrent refractory multiple myeloma (RRMM).

Sensitivity analysis: The sensitivity analysis was conducted by excluding individual studies one by one.

Language: English.

Country(ies) involved: China.

Keywords: Daratumumab; Multiple myeloma; CD38; Meta-analysis; Systematic review. 
Contributions of each author:

Author 1 - Yin Wang is responsible for the analysis of relevant materials and the writing of article.

Email: wangyin19@lzu.edu.cn

Author 2 - Yanqing $\mathrm{Li}$ is responsible for the data collection and assembly.

Email: 1464443255@qq.com

Author 3 - Ye Chai designe, instructe and revise article.

Email: papillon2009@163.com 